\title{
Malignant Findings in Candidates for Transcatheter Aortic Valve Implantation
}

\author{
Hiroshi Kadowaki, MD, Kazuyuki Yahagi, MD, Yu Horiuchi, MD, Kengo Tanabe, MD, PhD
}

Mitsui Memorial Hospital, Department of Cardiovascular Medicine, Tokyo, Japan

\section{ABSTRACT}

Background: In candidates for transcatheter aortic valve implantation (TAVI), preoperative computed tomography (CT) may detect clinically relevant non-cardiac findings. In particular, when malignant findings are detected, patients may be less likely to undergo the procedure. Additionally, they might require further examinations, which may prolong their time to treatment. We investigated how malignant findings affect candidacy for TAVI.

Methods: In this single-center retrospective study, 98 patients with severe aortic stenosis who had undergone preoperative CT between September 2013 and October 2016 were evaluated for malignant findings.

Results: Seven patients (7.1\%) had malignant findings. 74 of 91 patients who did not have malignant findings underwent TAVI, SAVR, or balloon aortic valvuloplasty (81.3\%). All patients who had malignant findings underwent TAVI or SAVR, and they underwent the procedure sooner after CT than the rest of the patients (mean time to TAVI or SAVR: $24.6 \pm 16.8$ versus $48.5 \pm 45.4$ days; $P=.003$ ). All 5 patients who had malignant findings without metastatic cancer and who underwent TAVI were still alive during the follow-up period (the mean duration of the follow-up period was $22.3 \pm$ 8.8 months). However, 1 patient who had a malignant finding with metastatic cancer died 7 months after CT.

Conclusion: Our outcomes indicated that the mean duration before TAVI or SAVR was reduced when malignant findings were detected by CT; and TAVI may be a safe and effective treatment for patients with aortic stenosis and a malignant tumor.

\section{INTRODUCTION}

In candidates for transcatheter aortic valve implantation (TAVI), computed tomography (CT) is necessary for valve sizing [Schultz 2010], considering the access site, and evaluating the perioperative risk [Latsios 2017]. However, preoperative CT may detect clinically relevant non-cardiac findings before TAVI. Several papers have shown that a certain percentage $(3.8 \%-8.7 \%)$ of patients with aortic stenosis are

Received August 5, 2019; received in revised form March 2, 2020; accepted March 13, 2020.

Correspondence: Hiroshi Kadowaki, MD, Mitsui Memorial Hospital, 1 KandaIzumicho, Chiyoda-ku, Tokyo 101-8643, Japan; +81-3-3862-9111; fax: +813-5687-9765 (e-mail: goota0626@gmail.com). incidentally found to have malignant findings (MFs) on CT before TAVI [Stachon 2015; Gufler 2014; Ben-Dor 2010; Goitein 2015]. Guidelines do not recommend TAVI or surgical aortic valve replacement (SAVR) for patients with severe AS whose life expectancy is less than 1 year [Nishimura 2014; Vahanian 2012]. We hypothesized that when CT detects MFs in patients with severe aortic stenosis (AS), they are less likely to be treated by TAVI or SAVR. Additionally, when CT detects MFs in candidates for TAVI, the time to TAVI or SAVR could be prolonged because of necessity for additional examinations and decision-making processes. Moreover, the life expectancy of patients with MFs could be shortened compared to those without MFs. Therefore, the aim of this study was to evaluate how MFs affect candidacy for TAVI.

\section{MATERIALS AND METHODS}

\section{Subjects and Study Design}

This was a single-center, retrospective study. 103 patients who considered TAVI in our hospital were identified as candidates for TAVI between September 2013 and October 2016 in Mitsui Memorial Hospital. We excluded 2 patients because of the severity of AS (moderate AS) and 3 patients because they already underwent CT at other hospitals. All patients who planned for TAVI underwent CT (cardiac CT

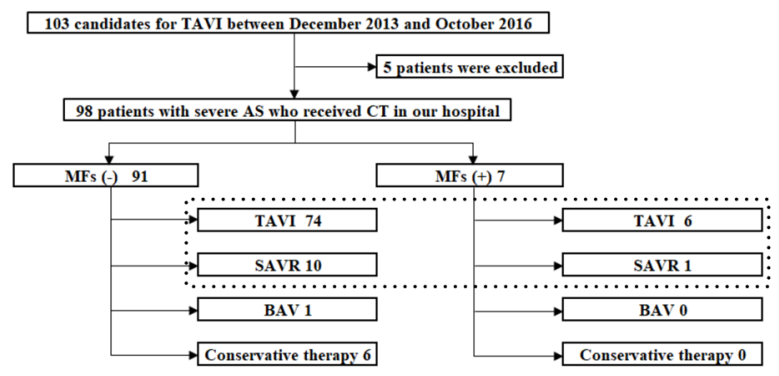

Flow diagram of patients with AS who were considered for TAVI in the present study. The duration from CT to invasive therapy (TAVI or SAVR) was calculated, and the mean duration before TAVI or SAVR is significantly reduced in the MFs $\left({ }^{+}\right)$group compared with that in the MFs (-) group (mean time to TAVI or SAVR: $24.6 \pm 16.8$ versus 48.5 \pm 45.4 days; $P=.003$ ) (dotted box). AS indicates aortic stenosis; CT, computed tomography; MF, malignant finding; TAVI, transcatheter aortic valve implantation; SAVR, surgical aortic valve replacement; BAV, balloon aortic valvuloplasty. MFs (-), malignant findings (-); MFs (+), malignant findings $(+)$. 
and whole-body CT) and then received TAVI, SAVR, balloon aortic valvuloplasty (BAV), or conservative therapy.

The method of treating severe AS was decided by consensus after discussion among a heart team (including cardiologists, cardiac surgeons, a medical engineer, nurse, and others). Especially when malignant findings were detected by CT, treatment strategies were carefully determined in close collaboration between a heart team and oncologists, considering the merit of each patient, ethical values, and current guidelines. The surgical risk was considered based on the patient's Society of Thoracic Surgeons (STS) score and Logistic EuroSCORE, which were calculated during the physician's assessment of the patient. When malignancies could be treated aggressively after TAVI and the patient had a life expectancy of more than 1 year with treatment, we performed TAVI. Patients who were considered ineligible for the invasive therapy received conservative therapy.

We divided the patients into two groups: MFs (+) or MFs $(-)$. We then investigated the clinical features of the patients in each group and the duration from CT to invasive therapy in order to determine how MFs affect candidacy for TAVI.

The participants provided informed consent, and the study was approved by an institutional review board.

\section{Assessment}

CT data were acquired with a 320-slice multi-detector row computed tomography scanner (Aquilion One, Canon Medical Systems Corporation, Otawara, Japan). All scans were analyzed by one experienced radiologist and reviewed by two cardiologists. The number of detected non-cardiac findings and primary MFs in each organ was counted. In this study, NYHA classification of each patient at baseline was determined just before TAVI procedure, not upon hospitalization.

\section{TAVI Procedure and Postoperative Medication}

TAVI was performed using the Edwards Sapien XT or Sapien 3 (Edwards Lifesciences, Irvine, CA, USA), or Corevalve (Medtronic, Minneapolis, MN, USA). The size of the prosthesis $(20,23,26$, or $29 \mathrm{~mm})$ was determined using echocardiography and CT before TAVI. Patients underwent TAVI by the transapical, transfemoral, or transsubclavian approach.

We usually administered dual antiplatelet therapy (i.e., aspirin and clopidogrel) for approximately 30 days following TAVI, as previously reported [Leon 2010]. In patients with MFs, the dosage schedule was modified individually in accordance with the bleeding risk due to the malignancy itself or the operative procedure. If patients needed to undergo operation within 30 days, antiplatelet therapy was stopped, and they underwent an operation for malignancy after the washout period with heparinization.

\section{Statistical analysis}

Clinical data are expressed as mean values and standard deviations. Data of the two groups were compared using Wilcoxon rank-sum test and Pearson chi-square test. A $P$ value $<.05$ was considered significant. All analyses were performed using JMP 14 (SAS Institute, Cary, NC, USA).

\section{RESULTS}

\section{Clinical Data}

Primary MFs were detected in 7 of 98 (7.1\%) patients. Regarding the baseline characteristics of the 98 patients, there was no remarkable difference between both groups except for the STS score and Logistic EuroSCORE (Table 1). The STS score was significantly lower in the MFs (+) group than in the MFs (-) group. The Logistic EuroSCORE tended to be lower in the MFs (+) group than in the MFs (-) group. No patient in either group had a medical history of malignancy.

The details of non-cardiac findings and MFs in each organ are summarized in Table 2. 74 of 98 (65.3\%) patients had non-cardiac findings. The detected MFs were lung, breast, pancreatic, colon, renal, urothelial, and bladder carcinoma. Subsequently, these patients were proven to have a primary malignant tumor. Findings other than MFs were detected and included interstitial pneumonia in 5 patients $(5.1 \%)$, abdominal aortic aneurysm in 6 patients $(6.1 \%)$, and liver cirrhosis in 1 patient (1\%).

\section{Treatment}

Among 98 severe AS patients who required treatment, MFs were detected in 7 patients. In the MFs (+) group, 6 patients underwent TAVI, 1 patient underwent SAVR, and zero patients underwent $\mathrm{BAV}$ and conservative therapy (Figure). 91 patients did not have MFs. 74 patients underwent TAVI, 10 patients underwent SAVR, 1 patient underwent $\mathrm{BAV}$, and 6 patients underwent conservative therapy in the MFs (-) group.

\section{Clinical Features of Patients with Malignant Findings}

Three patients in the MFs (+) group underwent TAVI and then an operation for malignancy. A 75 -year-old man had lung carcinoma and underwent TAVI 8 days after CT. The operation for malignancy was performed 56 days after CT. He was still alive at 27 months following CT. An 85-year-old woman had colon carcinoma and underwent TAVI 15 days after CT. The operation for malignancy was performed 27 days after CT. She was still alive at 28 months following CT. A 74-yearold woman had urothelial carcinoma and underwent TAVI 18 days after CT. The operation for malignancy was performed 52 days after CT. She was still alive at 32 months following $\mathrm{CT}$. The mean duration between CT and the operation for malignancy was $45.3 \pm 12.4$ days. All 3 patients who underwent TAVI and additional surgery were still alive during the follow-up period (the mean duration of the follow-up period was $29.0 \pm 2.2$ months) after CT.

Another 3 patients in the MFs (+) group underwent TAVI, but they did not undergo operation for malignancy. An 86-year-old man had pancreatic carcinoma and underwent TAVI 8 days after CT. However, he decided not to undergo further surgery after treatment of AS. He was still alive at 14 months CT. An 84-year-old woman had renal carcinoma and underwent TAVI 26 days after CT. She received imaging examinations regularly. She was still alive at 26 months following CT before TAVI. A 70-year-old woman had breast carcinoma and underwent TAVI 45 days after CT. 
Unfortunately, she had cancer metastasis to her right lung; however, she was expected to survive for more than 2 years when she received chemotherapy after TAVI. She was the only patient who had cancer metastasis in the MFs (+) group. She received hormone therapy following TAVI, but she eventually

Table 1. Baseline Characteristics of the 98 Patients

\begin{tabular}{|c|c|c|c|}
\hline & \multicolumn{2}{|c|}{ Malignant Findings } & \multirow{2}{*}{$\begin{array}{c}P \\
(-) \text { vs (+ }\end{array}$} \\
\hline & $(-)$ & $(+)$ & \\
\hline $\mathrm{n}$ & 91 & 7 & 98 \\
\hline Age & $83.6 \pm 5.8$ & $79.1 \pm 5.1$ & .06 \\
\hline Female sex & $65(71.4)$ & $3(42.9)$ & .2 \\
\hline Body weight, $\mathrm{kg}$ & $51.3 \pm 13.6$ & $54.3 \pm 10.8$ & .58 \\
\hline Body surface area, $\mathrm{m}^{2}$ & $1.4 \pm 0.2$ & $1.5 \pm 0.2$ & .13 \\
\hline Presence of hypertension & $74(81.3)$ & $5(71.4)$ & .52 \\
\hline Presence of diabetes & $26(28.6)$ & $0(0.0)$ & .1 \\
\hline Current/former smoker & $26(28.6)$ & $4(57.1)$ & .11 \\
\hline Presence of dyslipidemia & $63(69.2)$ & $3(42.9)$ & .14 \\
\hline Creatinine level, $\mathrm{mg} / \mathrm{dL}$ & $0.83 \pm 0.4$ & $1.08 \pm 0.4$ & .19 \\
\hline STS score & $5.7 \pm 2.9$ & $3.6 \pm 1.6$ & .03 \\
\hline Logistic EuroSCORE & $11.4 \pm 6.4$ & $7.0 \pm 3.0$ & .05 \\
\hline NYHA class & $2.2 \pm 0.7$ & $2.4 \pm 0.7$ & $.20 *$ \\
\hline \multicolumn{4}{|l|}{ Type of coronary artery disease } \\
\hline OMI & $9(9.9)$ & $0(0)$ & .38 \\
\hline $\mathrm{p}-\mathrm{PCl}$ & $19(20.9)$ & $1(8)$ & .68 \\
\hline $\mathrm{p}-\mathrm{CABG}$ & $9(9.9)$ & $1(8)$ & .71 \\
\hline Presence of cerebrovascular disease & $4(4.4)$ & 0 & .57 \\
\hline Presence of peripheral vascular disease & $4(4.4)$ & 0 & .57 \\
\hline \multicolumn{4}{|l|}{ Type of atrial fibrillation } \\
\hline Chronic (\%) & $13(14.3)$ & 0 & .28 \\
\hline Paroxysmal (\%) & $11(12.0)$ & 0 & .33 \\
\hline \multicolumn{4}{|l|}{ Echocardiographic findings } \\
\hline Aortic valve area, $\mathrm{cm}^{2}$ & $0.63 \pm 0.1$ & $0.73 \pm 0.1$ & .07 \\
\hline Mean PG, $\mathrm{mmHg}$ & $55.1 \pm 20.1$ & $52.7 \pm 11.8$ & .8 \\
\hline Moderate or severe AR & $9(9.9)$ & $0(0.0)$ & .38 \\
\hline Moderate or severe MR & $11(12.0)$ & $2(28.6)$ & .47 \\
\hline$<$ Ejection fraction (\%) & $66.4 \pm 12.2$ & $68.6 \pm 10.1$ & .65 \\
\hline Presence of COPD (\%) & $7(7.7 \%)$ & $1(14.3)$ & .45 \\
\hline
\end{tabular}

Continuous data are presented as mean $\pm \mathrm{SD}$ and categorical data as number (\%). *No statistic validity because of the small sample size. SD indicates standard deviation; STS, Society of Thoracic Surgeons; NYHA, New York Heart Association; OMI, old myocardial infarction; $\mathrm{p}-\mathrm{PCl}$, post-percutaneous coronary intervention; $\mathrm{p}-\mathrm{CABG}$, post coronary artery bypass graft; $\mathrm{PG}$, pressure gradient; $A R$, aortic regurgitation; $M R$, mitral regurgitation; COPD, chronic obstructive pulmonary disease. died of infection at 7 months following CT. A 76-year-old man with bladder carcinoma (stage 0a) underwent SAVR and

Table 2. Non-cardiac and Malignant Findings in Each Organ

$\begin{array}{lr}\text { Lung } & \\ \text { Lung carcinoma } & 1 \\ \text { Emphysema } & 7 \\ \text { Atelectasis } & 3 \\ \text { Interstitial pneumonia } & 5 \\ \text { Cancer metastasis } & 1 \\ \text { Aorta } & 3 \\ \text { Aortic dilatation } & 3 \\ \text { Aneurysm } & 1 \\ \text { Breast } & 1 \\ \text { Breast carcinoma } & \\ \text { Gynecomastia } & 6 \\ \text { Thyroid } & 1 \\ \text { Cyst } & 3 \\ \text { Liver } & 2 \\ \text { Liver Cirrhosis } & 10 \\ \text { Fatty liver } & \\ \text { Hemangioma } & \\ \text { Cyst } & \end{array}$

Pancreas

Pancreatic carcinoma

Pancreatic calculus

Gall bladder

Cholelith 10

Enlargement 2

Extraction 1

Intestine

Colon carcinoma

Colon diverticulum 1

Kidney

Renal cell carcinoma

Hydronephrosis

Cyst $\quad 24$

Ureter

Urothelial carcinoma $\quad 1$

Prostate

Prostatic hypertrophy 1

Bladder

Bladder carcinoma $\quad 1$ 
mitral valve replacement of the existence of moderate mitral regurgitation.

\section{Duration from CT to Invasive Therapy and Prognosis after TAVI}

The duration from CT to invasive therapy (TAVI or SAVR) was calculated, and the mean duration before TAVI or SAVR was significantly reduced in the MFs (+) group compared with that in the MFs (-) group (mean time to TAVI or SAVR: 24.6 \pm 16.8 versus $48.5 \pm 45.4$ days; $P=.003$ ) (dotted box in Figure). Moreover, among patients who underwent TAVI without metastatic cancer, 5 of 6 patients (83.3\%) were still alive during the follow-up period (the mean duration of the follow-up period was $22.3 \pm 8.8$ months). The patient who had cancer metastasis died at 7 months following CT before TAVI.

\section{Complications}

Major complications such as stroke, hemorrhage, conduction abnormalities, clinically relevant paravalvular leak, or infective endocarditis did not occur within 30 days.

\section{DISCUSSION}

\section{Probability of Non-Cardiac and Malignant Findings by CT in Candidates for TAVI}

In the present study, 64 of 98 patients (65.3\%) had noncardiac findings and 7 of 98 patients (7.1\%) had malignant findings. It was previously reported that non-cardiac findings were identified in $58.1 \%$ of 503 consecutive patients with suspected coronary artery disease who underwent diagnostic cardiac multiple detector CT [Onuma 2006]. In our study, CT detected non-cardiac findings with a higher probability in candidates for TAVI. In addition, the probability of lifethreatening diseases, such as interstitial pneumonia, abdominal aortic aneurysm, and cirrhosis of the liver was higher than previously described [Onuma 2006]. This finding may be because more TAVI candidates are elderly individuals. Cardiologists must interpret CT scans carefully and consider cardiac and non-cardiac findings because non-cardiac findings can affect the clinical course of patients who are candidates for TAVI.

MFs may especially affect the clinical course more remarkably. In patients with severe AS, the frequency of MFs in our hospital was $7.1 \%$, which was similar to that in previous reports [Stachon 2015; Gufler 2014; Ben-Dor 2010; Goitein 2015]. MFs cannot be ignored even in a few percent. Thus, cardiologists should pay attention to non-cardiac findings and MFs detected by CT before TAVI.

\section{How Malignant Findings Affect Candidacy for TAVI}

In patients who are scheduled for elective surgery, aortic valve replacement is recommended in symptomatic severe AS in accordance with the European Society of Cardiology and the European Society of Anesthesiology guidelines [Kristensen 2014]. Therefore, patients who have symptomatic severe AS or who are scheduled for high-risk surgery need to be treated for AS first. Furthermore, we performed TAVI to avoid extracorporeal circulation during SAVR from the perspective of depression of cellular immunity [Markewitz 1996; Hisatomi 1989] and because TAVI is associated with minimal invasiveness and a short recovery time [Merkel 2015]. Patients with MFs in our study needed to undergo TAVI or SAVR before receiving treatment for cancer. We considered performing BAV in such a situation, but BAV is associated with risks of complications including bleeding, cerebrovascular events, and others. Therefore, we considered TAVI first, not BAV [Hara 2007].

Consequently, all patients with AS and MFs underwent invasive therapy (TAVI or SAVR). Because of the existence of moderate mitral regurgitation, one patient underwent SAVR and mitral valve replacement. A previous study reported that the appearance of potentially malignant incidental findings did not significantly affect the therapeutic decisions [Stachon 2015]. However, the number of patients with cancer metastasis in this study was larger than that of our study. On the other hand, there were few metastatic findings in our study. The difference between these studies may be caused by the existence of metastatic cancer. In other words, there is a possibility that the decision of how to treat severe AS in candidates for TAVI depends on whether the patient has metastatic cancer, not on the existence of cancer.

When the treatment strategies for AS were decided, we performed treatment of AS as soon as possible because we thought that a short duration between CT and the operation would achieve a better prognosis of malignancy with AS, and immediate treatment led to a significantly reduced mean duration before TAVI or SAVR in the MFs (+) group over the MFs (-) group. Furthermore, the duration from CT to invasive therapy (TAVI or SAVR) in the MFs (+) group was remarkably short compared to that in a previous report (mean time to TAVI or SAVR: $24.6 \pm 16.8$ and $91 \pm 152$ days, respectively) [Stachon 2015].

\section{Effectiveness of TAVI}

In patients who underwent TAVI, 5 of 6 patients (83.3\%) were still alive during the follow-up period (the mean duration of the follow-up period was $22.3 \pm 8.8$ months). Considering the coexistence of malignant tumors and severe AS, these patients might have a comparatively better prognosis than those who did not undergo TAVI and an operation for malignancy [Okura 2018; Schechter 2019]. Further, major complications did not occur within 30 days. This outcome was better than that of the patient group that was strongly suspected to have a malignant tumor and cancer metastasis [Stachon 2015]. In addition, all 3 patients who underwent TAVI and additional surgery were still alive during the follow-up period after CT. We think this prognosis is one resulting from performing TAVI earlier after CT and conducting an operation for cancer rapidly following TAVI. TAVI may be an effective treatment for patients with AS and a malignant tumor.

A history of malignancy has been already reported to be associated with poor prognosis in patients with severe AS [Minamino-Muta 2018]. There is a possibility that the lack of patients with history of malignancy in the MFs (+) group led to the better prognosis. Another study reported that cancer 
metastasis was one of the independent predictors of shorter survival in patients with TAVI in a multivariable Cox proportional hazard regression analysis of 749 patients undergoing TAVI [Watanabe 2016]. In fact, only 1 patient in our study with metastatic breast cancer, whose life expectancy had been more than 2 years and who underwent TAVI for severe AS, died of infection within 7 months. Therefore, the favorable prognosis in our study may have resulted from the small number of patients with metastatic cancer. However, this is only speculation given that the present study is a single institution study with a small number of patients.

In recent years, the survival rate of patients with cancer has improved worldwide [Allemani 2018], and the number of TAVIs performed has increased [O'Sullivan 2017]. The probability and effect of malignant CT findings on candidacy for TAVI will become a more serious problem in daily clinical practice, so further large-scale clinical research is needed on this topic.

\section{Study Limitations}

This study has several limitations. First, MFs (+) patients may have tended to undergo TAVI because of the lower STS score and Logistic EuroSCORE. Second, the favorable prognosis in our patients with severe AS and cancer might have been because of the small proportion of patients with metastatic cancer. Third, this study was not randomized. Therefore, any potential benefits of TAVI compared to SAVR, BAV, and medical therapy are unclear. The effect of MFs on candidacy for TAVI needs to be investigated further.

\section{REFERENCES}

Allemani C, Matsuda T, Di Carlo V, et al. 2018. Global surveillance of trends in cancer survival 2000-14 (CONCORD-3): analysis of individual records for 37513025 patients diagnosed with one of 18 cancers from 322 population-based registries in 71 countries. Lancet 391:1023-75.

Ben-Dor I, Waksman R, Hanna NN, et al. 2010. Utility of radiologic review for noncardiac findings on multislice computed tomography in patients with severe aortic stenosis evaluated for transcatheter aortic valve implantation. Am J Cardiol 105:1461-4.

Goitein O, Di Segni E, Eshet Y, et al. 2015. Non-valvular findings before trans-catheter aortic valve implantation and their impact on the procedure. Isr Med Assoc J 17:764-7.

Gufler H, Schulze CG, Wagner S. 2014. Incidental findings in computed tomographic angiography for planning percutaneous aortic valve replacement: advanced age, increased cancer prevalence? Acta Radiol 55:420-6.

Hara H, Pedersen WR, Ladich E, et al. 2007. Percutaneous balloon aortic valvuloplasty revisited: time for a renaissance? Circulation 115:e334-8.

Hisatomi K, Isomura T, Kawara T, et al. 1989. Changes in lymphocyte subsets, mitogen responsiveness, and interleukin-2 production after cardiac operations. J Thorac Cardiovasc Surg 98:580-91.

Kristensen SD, Knuuti J, Saraste A, et al. 2014. 2014 ESC/ESA Guidelines on non-cardiac surgery: cardiovascular assessment and management: the Joint Task Force on non-cardiac surgery: cardiovascular assessment and management of the European Society of Cardiology (ESC) and the European Society of Anaesthesiology (ESA). Eur Heart J 35:2383-431.

Latsios G, Spyridopoulos TN, Toutouzas K, et al. 2017. Multi-slice CT (MSCT) imaging in pretrans-catheter aortic valve implantation (TAVI) screening. How to perform and how to interpret. Hellenic J Cardiol 59:3-7.

Leon MB, Smith CR, Mack M, et al. 2010. Transcatheter aortic-valve implantation for aortic stenosis in patients who cannot undergo surgery. N Engl J Med 363:1597-607.

Markewitz A, Faist E, Lang S, Hültner L, Weinhold C, Reichart B. 1996. An imbalance in T-helper cell subsets alters immune response after cardiac surgery. Eur J Cardiothorac Surg 10:61-7.

Merkel S, Eikermann M, Neugebauer EA, von Bandemer S. 2015. The transcatheter aortic valve implementation (TAVI) - a qualitative approach to the implementation and diffusion of a minimally invasive surgical procedure. Eur Heart J Cardiovasc Imaging. 16:731-7.

Minamino-Muta, Kato T, Morimoto T, et al. 2018. Malignant disease as a comorbidity in patients with severe aortic stenosis: clinical presentation, outcomes, and management. Eur Heart J Qual Care Clin Outcomes 4:180-8.

Nishimura RA, Otto CM, Bonow RO, et al. 2014. 2014 AHA/ACC guideline for the management of patients with valvular heart disease: executive summary: a report of American college of Cardiology/American Heart Association Task Force on Practice Guidelines. J Am Coll Cardiol 63:2438-88.

Okura Y, Ishigaki S, Sakakibara S, et al. 2018. Prognosis of cancer patients with aortic stenosis under optimal cancer therapies and conservative cardiac treatments. Int Heart J 59:750-8.

Onuma Y, Tanabe K, Nakazawa G, et al. 2006. Noncardiac findings in cardiac imaging with multidetector computed tomography. J Am Coll Cardiol 48:402-6.

O'Sullivan CJ, Wenaweser P. 2017. A glimpse into the future: in 2020, which patients will undergo TAVI or SAVR? Interv Cardiol 12:44-50.

Schechter M, Balanescu DV, Donisan T, et al. 2019 An update on the management and outcomes of cancer patients with severe aortic stenosis. Catheter Cardiovasc Interv 94:438-45.

Schultz CJ, Moelker AD, Tzikas A, et al. 2010. Cardiac CT: necessary for precise sizing for transcatheter aortic implantation. Euro Intervention 6 Suppl G:G6-13.

Stachon P, Kaier K, Milde S, et al. 2015. Two-year survival of patients screened for transcatheter aortic valve replacement with potentially malignant incidental findings in initial body computed tomography. Eur Heart J Cardiovasc Imaging 16:731-7.

Vahanian A, Alfieri O, Andreotti F, et al. 2012. Guidelines on the management of valvular heart disease (version 2012): the Joint Task Force on the Management of Valvular Heart Disease of the European Society of Cardiology (ESC) and the European Association for Cardio-Thoracic Surgery (EACTS). Eur Heart J 33:2451-96.

Watanabe Y, Kozuma K, Hioki H, et al. 2016. Comparison of results of transcatheter aortic valve implantation in patients with versus without active cancer. Am J Cardiol 118: 572-7. 